African Crop Science Journal by African Crop Science Society is licensed under a Creative Commons Attribution 3.0 Uganda License. Based on a work at www.ajol.info/ and www.bioline.org.br/cs DOI: https://dx.doi.org/10.4314/acsj.v27i3.2

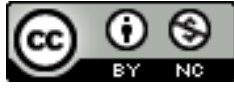

\title{
EFFET DES COMPOSANTES DE LA TECHNOLOGIE MICRODOSE SUR LA PERFORMANCE ÉCONOMIQUE ET FINANCIÈRE DES EXPLOITATIONS AGRICOLES DU KOURITENGA ET DU ZONDOMA AU BURKINA FASO
}

\author{
H. SIGUE ${ }^{1}$, I.A. LABIYI ${ }^{2}$, K. ISSAKA ${ }^{2,3}$, J.A. YABI ${ }^{2}$ et G. BIAOU ${ }^{4}$ \\ 'Institut de l'Environnement et de Recherches Agricoles (INERA), BP : 208, Fada N'Gourma, \\ Burkina Faso \\ ${ }^{2}$ Laboratoire d'Analyses et de Recherches sur les Dynamiques Economique et Sociale (LARDES), \\ Faculté d'Agronomie, Université de Parakou, BP : 123 Parakou, Bénin \\ ${ }^{3}$ Agence Territoriale pour le Développement Agricole (ATDA-Pôle 4), Parakou, Bénin \\ ${ }^{4}$ Université Nationale d'Agriculture (UNA), Porto-Novo, Bénin \\ Corresponding author : hamsig65@gmail.com
}

(Received 22 August 2018; accepted 30 July 2019)

\section{RÉSUMÉ}

Les processus naturels et de l'action anthropique ont des conséquences sur la baisse de la productivité des terres agricoles. C'est ainsi que les agriculteurs dans les localités qui sont affectées, mettent en œuvre diverses technologies de gestion des terres en vue d'améliorer la productivité. L'objectif de cette étude était d'évaluer l'effet des composantes de la technologie 'Microdose' sur la performance économique et financière des exploitations agricoles dans les localités de Kouritenga et Zondoma au Burkina Faso. Une enquête a été conduite auprès d'un échantillon de 360 ménages agricoles appliquant au moins une composante de la technologie sur un minimum de 0,5 ha. Les données sur les paramètres socio-économiques, les quantités et prix des intrants et des produits mis en jeu dans les composantes de la technologie, ont été collectées à travers un questionnaire semi structuré. Les indicateurs de rentabilité économique et financière ont été évalués et comparés selon les composantes, suivie d'une régression à équations simultanées pour déterminer les facteurs influençant la performance économique. Les résultats ont montré que la technologie 'Microdose $(M D)$ avec Bandes enherbées $(B E)$ avec Compost (CO)' présentait une meilleure performance du point de vue de la marge nette, du taux interne de rentabilité et du ratio bénéfice-coût. Cependant, l'adoption de la microdose a un effet positif et significatif sur la performance économique de production tandis que la technologie 'Microdose $(\mathrm{MD})$ avec Cordon pierreux $(\mathrm{CP})+$ Compost $(\mathrm{CO})$ a influencé négativement et significativement la performance économique des exploitations. Ces résultats obtenus pourraient servir à définir des axes de recherche et des stratégies d'intervention au profit des exploitants agricoles.

Mots Clés : Burina Faso, rentabilité économique et financière, exploitations agricoles, technologie Microdose 


\begin{abstract}
Natural processes and anthropogenic actions have consequences for the decline of agricultural land productivity. Farmers in affected communities implement a variety of land management technologies to improve productivity. The objective of this study was to evaluate the effect of Microdose technology components on the economic and financial performance of farms in the Kouritenga and Zondoma localities in Burkina Faso. A survey was conducted among a sample of 360 farm households applying at least one component of the technology for a minimum of 0.5 ha. Data on socio-economic parameters, quantities and prices of inputs and outputs involved in the components of technology were collected through a semi-structured questionnaire. Economic and financial performance indicators were evaluated and compared by component, followed by simultaneous equation regression to determine factors influencing economic performance. The results showed that 'Microdose (MD) with Grass Strips (BE) with Compost (CO)' showed better performance in terms of net margin, internal rate of return and benefit-cost ratio. The adoption of microdose has a positive and significant effect on the economic performance of production while the technology 'Microdose (MD) with Stonecord (CP) + Compost (CO) has negative and significantly influenced on the economic performance of farms. These results could be used to define lines of research and intervention strategies for the farmers benefit.
\end{abstract}

Key Words: Burina Faso, economic and financial profitability, farms, Microdose technology

\section{INTRODUCTION}

En Afrique subsaharienne, l'agriculture est à la base de l'économie et la principale source de revenu des ménages agricoles. Cette agriculture peine cependant à assurer les besoins d'une population de plus en plus croissante face à l'insécurité alimentaire. Depuis quelques années, on assiste à une régression globale des rendements des cultures, conséquences d'une baisse de la pluviométrie et du phénomène de la baisse de la fertilité des terres agricoles (Abossolo et al., 2017; Sorel et al., 2017). Ainsi, la baisse des rendements des cultures vivrières et de rente entraîne une utilisation non contrôlée et intensive des intrants agricoles (Amoussou et al., 2016).

Au Burkina, les provinces du Zondoma et du Kouritenga situées dans le plateau, les systèmes de production sont caractérisés par les cultures de mil, du sorgho, du maïs et du niébé. Un diagnostic d'évaluation participative conduite par l'INERA et ses partenaires (AGRA, 2010) a révélé un déclin des rendements des cultures et de la production globale ce qui a contribué à accroître l'insécurité alimentaire et nutritionnelle dans ces localités (Ouédraogo, 2012).

Pour pallier cette situation, les producteurs dans ces zones sont perpétuellement à la recherche de moyens susceptibles d'optimiser la production des cultures en limitant les coûts de production. Ils sont appuyés par l'Etat et ses partenaires à travers des projets et programmes. Des technologies agricoles ont été développées dont la diversité de stratégies de gestion et de conservation des sols avec l'application de la technologie "Microdose".

En effet, l'adoption d'une innovation fait référence à la décision de mettre en œuvre des propositions techniques nouvelles dans des systèmes de production existants et d'améliorer progressivement leur utilisation (Zakari, 2017). Ainsi, une invention ou un paquet technique peut être qualifié d'innovation quand il conduit à modifier un système de production de manière significative et durable. Ainsi, la technologie 'microdose est une combinaison de techniques de gestion de l'eau à la parcelle, de techniques de défense et de restauration des sols avec l'application des doses économiques d'engrais minéral au poquet. Elle présente des composantes basées 
sur l'option du producteur en fonction des moyens et du savoir -faire et surtout des objectifs de production.

Au Burkina Faso, les agriculteurs ont appris que la technologie 'Microdose' a un potentiel productif d'accroissement de la production et de génération de valeur ajoutée. Plusieurs années d'interventions des acteurs de développement du monde rural dans les provinces du Kouritenga et du Zondoma ont favorisé une vulgarisation de la technologie de 'Microdose'. Elle renferme plusieurs composantes (Microdose (MD) avec Cordons pierreux (CP) avec Compost (CO); Microdose $(M D)$ avec Cordons pierreux $(C P)$ avec Paillage (PA); Microdose (MD) avec Bandes enherbées (BE) avec Paillage $(P A))$ et l'adoption d'une telle innovation engendre des incertitudes supplémentaires (l'efficacité, le coût d'investissement, etc.) pour les agriculteurs qui s'ajoutent aux nombreux risques encourus dans le contexte de production.

Pour ce faire, cette étude vise à déterminer les composantes de la technologie 'Microdose' qui offrent les meilleures performances économiques dans les exploitations agricoles des deux localités de l'étude. Des indicateurs de revenu couramment utilisés fournissent des éléments sur la performance des exploitations. La valeur ajoutée nette d'exploitation par unité de travail/année donne une indication de ce qui est disponible pour rémunérer l'ensemble des facteurs terres, capitaux et main d'œuvre et un indicateur de revenu net (revenu de l'exploitant et sa famille par unité de travail familial) (McClintock et al., 1989). Le capital d'exploitation notamment les facteurs et plus particulièrement ceux qui contribuent à rendre la production plus efficace (matériel, équipements et intrants, etc.) sont plus faibles chez les ménages agricoles dans les localités expliquant la faiblesse des investissements.

Il n'est pas possible d'isoler des déterminants communs à toutes les situations, en raison notamment de l'hétérogénéité des agriculteurs et des contextes de production
(Knowler et Bradshaw, 2007; Prokopy et al., 2008; Baumgart-Getz et al., 2012).

Dans ce contexte, la question qui se pose est de savoir, quel est l'effet de la technologie 'Microdose' sur la performance économique des exploitations agricoles au Kouritenga et au Zondoma ? La pertinence de cette étude réside dans les connaissances élaborées relatives aux pratiques de la microdose avec les autres techniques culturales de gestion de l'eau et de conservation de la fertilité des sols en production vivrière dans les deux provinces.

\section{MATÉRIEL ET MÉTHODES}

Zone de l'étude. L'étude a été réalisée dans deux (02) provinces que sont le Zondoma localisé dans la région du Nord et le Kouritenga dans la région du Centre-Est. Ces provinces sont situées dans une zone climatique comprise entre les isohyètes 400 et $800 \mathrm{~mm} /$ an et correspondent à la zone d'intervention $\mathrm{du}$ projet INuWaM (Intégration des Nutriments et de la Gestion de l'eau pour une Agriculture Durable au Sahel). Ces localités sont dans une zone agro-écologiques nordsoudaniennes avec des systèmes de production relativement homogènes. Les principales cultures pratiquées sont le mil, sorgho, maïs et niébé qui sont confrontées à des contraintes similaires de faible productivité agricole. Le choix porté sur ces localités vient du fait qu'elles ont bénéficié déjà dans les années 1980 de la diffusion de technologies agricoles CES/ DRS (Conservation des Eaux et des Sols/ Dé fense et Restauration des Sols). Récemment entre 2010 et 2015, ces provinces ont bénéficié de l'intervention des projets de gestion intégrée de la fertilité, des tests de démonstration avec la micro dose, la gestion de l'eau et de la fertilité.

Le choix des communes a été effectué à partir du gradient Nord-Sud et suivant l'accessibilité de la localité.

Echantillonnage et base de données. L'échantillon de l'enquête compte 360 ménages 
agricoles appliquant au moins une composante de la technologie sur au moins 0,5 ha sur des cultures sorgho, mil, maïs et niébé. La collecte des données était effectuée auprès des producteurs sélectionnés dont la superficie d'application de la technologie est représentative e" à 0,5 ha. L'application de la technologie 'Microdose' sur des superficies inférieures n'étant pas considérées du fait que les pressions des activités sont moindres. Six (06) villages ont été retenus dans la zone : 03 villages par provinces à raison d'un village par commune (Tableau 1). Un total de 180 ont été tirés de façon aléatoire par province. Au total, 360 ménages adoptants et non adoptants ont constitué l'échantillon et ont effectivement été enquêtés.

Aussi, des entretiens de groupe ont été conduits auprès des groupes de personnes fortement impliquées dans la production agricole les paysans innovateurs. Ils ont permis d'identifier les formes d'adaptation et d'évaluer la récurrence des stratégies.

Les données de la présente étude sont issues d'une enquête de terrain dans les deux provinces, qui s'est déroulée en 2014.

L'enquête ménage a concerné les chefs de ménage adoptant le paquet technologique ou non dont les données collectées ont regroupé: (i) les caractéristiques socio-économiques des ménages et démographiques; et (ii) les indicateurs de performances des composantes de la technologie.

Approche théorique. Dans la littérature, la performance se rapporte à la fois à l'optimisation des moyens dans leur utilisation, mais aussi au pilotage des objectifs stratégiques. Elle est à la fois contingente aux objectifs fixés à atteindre, mais aussi fonction des destinataires de l'information (PlatetPierrot, 2009).

Dans un autre contexte, la méthode IDEA (Indicateurs de Durabilité des Exploitations Agricoles), rend compte du concept de performance globale d'une exploitation agricole. Avec cette méthode de performance globale, la productivité est perçue comme un indicateur de performance économique. La performance économique dans les exploitations agricoles signifie que la valeur marginale de produit par unité de dépenses des inputs doit être égale pour toutes les ressources dans toutes les activités agricoles. Ce qui définit les aspects technico-économiques de la productivité globale et exprime la performance économique de l'exploitation agricole.

La performance peut être utilisée pour comparer des exploitations de différentes structures économiques et on distingue

TABLEAU 1. Structure de l'échantillon de l'étude

\begin{tabular}{|c|c|c|c|c|c|}
\hline Provinces & Communes & Villages & Adoptants & $\begin{array}{c}\text { Non } \\
\text { adoptants }\end{array}$ & $\begin{array}{c}\text { Taille de } \\
\text { l'échantillon }\end{array}$ \\
\hline Kouritenga & $\begin{array}{l}\text { Andemtenga } \\
\text { Koupèla } \\
\text { Gounghin }\end{array}$ & $\begin{array}{l}\text { Songretenga } \\
\text { Ronsin } \\
\text { Kabeiga }\end{array}$ & 167 & 13 & $\begin{array}{l}60 \\
60 \\
60\end{array}$ \\
\hline Zondoma & $\begin{array}{l}\text { Lèba } \\
\text { Gourcy } \\
\text { Boussou }\end{array}$ & $\begin{array}{l}\text { Raguèguema } \\
\text { Kagapessogo } \\
\text { Boussou }\end{array}$ & 160 & 20 & $\begin{array}{l}60 \\
60 \\
60\end{array}$ \\
\hline Zone d'étude & - & - & 327 & 33 & 360 \\
\hline
\end{tabular}

Source : Enquête socio-économique, 2014 
généralement, la performance économique et la performance financière. Zahm (2011) et Zahm et Mouchet (2013) définissent la performance globale d'une exploitation agricole comme son niveau de contribution à une agriculture durable. Les agriculteurs s'intéressent aux critères permettant de qualifier leurs activités et des méthodes d'évaluation scientifiquement fondées pour rendre compte de leur performance individuelle ou collective vers une agriculture durable.

Dans un processus de production raisonnée, le producteur cherche avant tout à optimiser sous la contrainte de coûts de production l'utilisation des ressources, donc à maximiser son profit. Pour ce faire, il combine les facteurs de producteurs de manière à atteindre le meilleur niveau de performance économique qui selon la théorie économique néoclassique correspond au point d'équilibre entre les revenus marginaux et les coûts marginaux. Dans ce modèle trois composantes de l'utilisation optimale des ressources, fournissant les conditions d'une performance économique. De ce fait, quatre (04) indicateurs déterminant les conditions d'une performance économique ont été utilisés dans cette étude, à savoir : la marge nette; la productivité moyenne de la main d'œuvre familiale ; le taux interne de rentabilité et le ratio Bénéfice-coût. Par ailleurs, dans la littérature, il existe plusieurs méthodes d'évaluation de la performance économique d'un système de production. Il y a entre autres, la méthode basée sur le bilan ou celle utilisant le compte de résultat ou compte d'exploitation (Yegbemey et al., 2014), la méthode avant-après et la méthode avecsans.

Dans cet article, le fondement théorique est celui de l'approche «avec et sans » pour apprécier l'effet de la technologie 'Microdose' sur la performance économique et financière des exploitations agricoles. Plusieurs analyses ont utilisé cette approche pour l'évaluation de l'efficacité ou de la performance des technologies agricoles Yabi et al. (2012). A cet effet, l'analyse est faite suivante deux démarches. La première consiste à calculer et comparer les indicateurs de performance tandis que la deuxième repose sur un modèle de régression économétrique afin d'identifier les déterminants de performance.

Indicateurs de performance économique et financière. Dans cette démarche, plusieurs indicateurs sont généralement utilisés Paraiso et al. (2012). La productivité globale, la productivité moyenne de la main d'œuvre familiale (PML), la marge nette le ratio Bénéfice-Coût et le taux de rentabilité interne ont été utilisés pour comparer la performance économique.

La marge nette (MN), est obtenue en déduisant du produit brut en valeur, les coûts totaux de production ou en déduisant de la marge brute les coûts fixes (Paraiso et al., 2012).

$M N=P B-(C V+C F)$ ou $M N=M B-C F$

Avec :

CV : les coûts variables, correspondent aux dépenses liées à l'acquisition d'intrants (engrais, insecticides, pesticides et main d'œuvre) (en FCFA/ha);

CF: les coûts fixes correspondent aux dépenses faites par l'exploitation mais non liées au volume de production : ce sont principalement les dépenses d'investissement. Les CF sont déterminés en appliquant un taux d' amortissement linéaire à la valeur totale d'acquisition du matériel. Ce taux correspond à l'inverse de la durée de la vie de ce matériel.

Si $M N>0$, l'activité de production de est économiquement rentable. Le produit brut permet donc de couvrir toutes les charges de production, par contre si $M N<0$, l'activité n'est pas rentable du point de vue économique. 
La productivité moyenne du travail est donnée par la formule suivante :

$$
\mathrm{PML}=\frac{M N}{M O}
$$

Avec $P M L$ : productivité moyenne nette du travail (main d'œuvre familiale en FCFA/Hj). C'est la rémunération journalière du travail d'un actif adulte au sein de l'exploitation.

$M N$ : marge nette en FCFA/ha

MO : main d'œuvre familiale utilisée en $\mathrm{Hj} / \mathrm{ha}$

Si PML > p (avec $p$ le salaire journalier payé à un homme- dans les deux provinces), alors l'activité est rentable du point de vue salaire payé. Dans le cas contraire elle ne l'est pas.

Le taux de rentabilité économique interne représente la productivité du capital et peut être déterminé par la formule :

$$
T R I=\frac{M N}{C T+V M O}
$$

$C T$ : coûts totaux de production en FCFA/ ha ;

$V M O$ : le main d'œuvre familiale en valeur FCFA/ha. Il est déterminé en multipliant la quantité physique de la main d'œuvre par ha par cout d'opportunité de la main d'œuvre dans les provinces de Zondoma et Kouritenga

TRI : taux de rentabilité interne exprimé en $\%$.

Il mesure la marge nette par unité de capital investi. Dans ce cas, le capital investi correspond aux couts totaux de production (Yabi et al., 2012). Il permet également d'apprécier la capacité d'une exploitation à rentabiliser le capital. Il est interprété en comparant au taux d'intérêt $i$ appliqué par les institutions bancaires ou de micro finance au Zondoma et Kouritenga. Ainsi, si TRI > i, l'activité est économiquement rentable du point de vue de l'investissement. Si TRI < i, l'activité n'es pas rentable du point de vue de l'investissement.

Le Ratio Bénéfice/Coût ou B/C exprime le gain financier total obtenu par l'investissement d'une unité monétaire (1 franc cfa par exemple). Soit B l'ensemble des bénéfices obtenus après un investissement total C. Il vient alors que :

$$
B / C=\frac{B}{C}
$$

En économie agricole, B est désigné par le produit brut obtenu en valeur et $\mathrm{C}$ par l'ensemble de tous les coûts exprimés en valeur, y compris la main d'œuvre familiale. Ainsi, si $P B V$ est le produit brut en valeur, les coûts totaux qui ne prennent pas en compte la valeur de la main-d'œuvre familiale et la valeur totale de la main-d'œuvre familiale utilisée, on a :

$$
B / C=\frac{P B V}{(C T+M O V)}
$$

MOV: Dans la pratique, est obtenu en multipliant la quantité physique de maind'œuvre familiale totale par le prix moyen de la main-d'œuvre salariée dans la zone de l'étude.

\section{Spécification du modèle de déterminants de} la performance des exploitations agricoles. L'analyse de l'effet de la technologie 'Microdose' sur la performance économique des exploitations agricoles repose sur les exploitants ayant adopté la technologie. Cela suppose que c'est seulement si la technologie est adoptée qu'il est possible d'analyser son effet. A cet effet, on peut se focaliser sur 
l'hypothèse selon laquelle la rentabilité économique et financière d'une exploitation agricole est déterminée par les caractéristiques socio-économiques du producteur, ses capitaux mais aussi dans le cadre de cette étude, par ses stratégies de restauration du sol, donc la technologie 'Microdose'.

Estimer les déterminants de la performance par les Moindres Carrés Ordinaire (MCO) par des régressions linéaires simples, comporte de biais d'endogénéité. Pour cela, admettons que la probabilité pour qu'une exploitation agricole soit rentable ou non à partir du résultat de sa PML, de son TRI ou B/C est corrélée avec la probabilité pour qu'elle le soit ou non à partir de sa MN. Partant de ces considérations, les déterminants du niveau de rentabilité des exploitations agricoles ont été appréciés par la modélisation impliquant un ensemble d'équations qui peuvent être reliées non seulement parce qu'elles agissent l'une sur l'autre mais aussi parce que leurs termes d'erreurs sont corrélés (Yegbemey et al., 2014). L'efficacité de cette estimation repose sur la valeur du coefficient de corrélation Chicarré. En effet, lorsque celui-ci est proche de 0 , l'estimation des coefficients de l'équation substantielle par les MCO n'est pas biaisée.

Ainsi, la performance économique et financière (P) de production du maïs, du mil et sorgho sont fonction des caractéristiques socioéconomiques des producteurs $(Z)$, leurs capitaux (C), et leurs composantes technologiques de 'Microdose' (M).

Soit la formulation suivante :

$$
P=f(Z, C, M)
$$

Etant donné que cette étude emploie quatre (04) indicateurs différents de la rentabilité, nous pouvons imposer à $\mathrm{P}$ un vecteur de $\mathrm{k}$ variables (4) de performance, un vecteur de $\mathrm{j}$ variables les caractéristiques socioéconomiques des producteurs. Ceci étant, nous avons $\mathrm{Z}$ comme un vecteur de $\mathrm{kJ} \times 1$ caractéristiques socio-économiques. Avec de

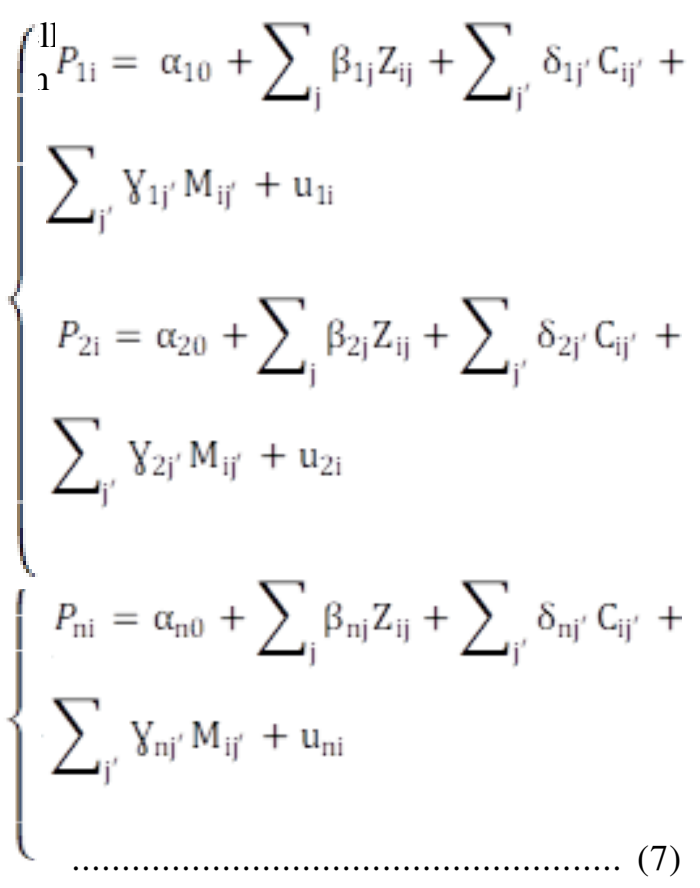

Dans ce système, les $\mathrm{P}_{1 \mathrm{n}}$ sont les indicateurs de la rentabilité (MN, PML, TRI et B/C respectivement); $\mathrm{a}_{0 \mathrm{n}}$ les limites constantes; le $\hat{a}_{j}$ est le coefficient de $S_{j}$ et les limites d'erreurs. Les coefficients á, â et "ont été estimés en utilisant la méthode d'OLS (moindre carré ordinaire) par une régression apparemment indépendante (SÛRE). De ces coefficients et de leurs niveaux d'importance, les facteurs affectant le niveau de la rentabilité économique de la production d'anacarde ont été déduits. L'ensemble de variables explicatives éventuelles $\mathrm{S}$ sont présentés dans le Tableau 2.

Les caractéristiques des variables explicatives pour les analyses sont consignées dans le Tableau 2.

Dans cette étude, la technologie 'Microdose' combine les techniques de conservation de l'eau, de gestion et les techniques de gestion de fertilité des sols. Dans le contexte de notre étude, la technologie Microdose est la pratique de l'une des composantes suivantes diffusées dans la zone d'étude: 
TABLEAU 2. C aractéristiques des variables explicatives pour les analyses

\begin{tabular}{|c|c|c|c|}
\hline Paramètres & Types de variables & Modalités & Signes attendus \\
\hline \multicolumn{4}{|c|}{ Caractéristiques socio-démographiques } \\
\hline Age & Variable continue & - & + \\
\hline Appartenance Association & Variable discontinue & $0=$ Non $; 1=$ Oui & - \\
\hline Statut matrimonial (marié) & Variable discontinue & $0=$ Non $; 1=$ Oui & + \\
\hline Sexe & Variable discontinue & $0=$ féminin $; 1=$ masculin & - \\
\hline Formation technique agricole & Variable discontinue & $0=$ Non $; 1=$ Oui & + \\
\hline Taille ménage & Variable continue & - & - \\
\hline Nombre d'actifs ménage & Variable continue & - & - \\
\hline Expérience en microdose & Variable discontinue & - & + \\
\hline Localité Kouritenga & Variable discontinue & $0=$ Non $; 1=$ Oui & $+/-$ \\
\hline Localité Zondoma & Variable discontinue & $0=$ Non $; 1=$ Oui & $+/-$ \\
\hline \multicolumn{4}{|c|}{ Capitaux } \\
\hline Charges fixes & Variable continue & - & - \\
\hline Charges variables & Variable continue & - & - \\
\hline Quantité de main d'œuvre totale & Variable continue & - & - \\
\hline \multicolumn{4}{|c|}{ Technologie microdose } \\
\hline Adoption microdose & Variable discontinue & $0=$ Non $; 1=$ Oui & + \\
\hline Cordon pierreux + Compost & Variable discontinue & $0=$ Non $; 1=$ Oui & - \\
\hline Bande enherbée + Paillage & Variable discontinue & $0=$ Non $; 1=$ Oui & - \\
\hline Bande enherbée + Compost & Variable discontinue & $0=$ Non $; 1=$ Oui & - \\
\hline Cordon pierreux + fumier & Variable discontinue & $0=$ Non $; 1=$ Oui & - \\
\hline
\end{tabular}


(i) Microdose (MD) avec Cordons pierreux (CP) avec Compost (CO);

(ii) Microdose (MD) avec Cordons pierreux (CP) avec Fumure organique (FO);

(iii) Microdose (MD) avec Cordons pierreux (CP) avec Paillage (PA);

(iv) Microdose (MD) avec Bandes enherbées (BE) avec Compost (CO);

(v) Microdose (MD) avec Bandes enherbées (BE) avec Fumure Organique (FO);

(vi) Microdose (MD) avec Bandes enherbées (BE) avec Paillage (PA);

(vii) Microdose (MD) avec Demi-lunes (DL) avec Compost (CO);

(viii) Microdose (MD) avec Demi-lunes (DL) avec Fumure organique (FO);

(ix) Microdose (MD) avec Zaï avec Compost (CO);

(x) Microdose (MD) avec Zaï avec Fumure organique (FO).

L'approche utilisée est basée sur l'évaluation des indicateurs de rentabilité économique et financière des exploitations agricoles avec l'estimation des effets au moyen d'une régression à équations simultanées avec le logiciel STATA 14. Une analyse comparée des résultats des indicateurs de performance a été effectuée entre les indicateurs et les composantes de la technologie.

\section{RÉSULTATS}

Caractéristiques socio-économique et démographique des producteurs. Le Tableau 3 présente les caractéristiques socio démographiques des répondants dans la zone d'étude.

Les adoptants de la technologie 'Microdose' étaient en majorité des hommes $(73,9 \%)$ qui cultivent le principalement le sorgho, le mil, le maïs et le niébé sur plus de 4 ha. Par ailleurs, moins de la moitié (41\%) de ces producteurs étaient en groupement ou association dont $23 \%$ ont reçu une formation en technologie 'Microdose' dans la zone d'étude.
Dans l'ensemble, les exploitants enquêtés avaient un âge compris entre 18 à 75 ans avec une moyenne de 45 ans. Ceux ayant entre 30 et 65 ans sont majoritaires (86\%) et sont en majorité des hommes $(65 \%)$. Ainsi, la moitié des chefs d'exploitation $(50,3 \%)$ étaient monogames contre $46,9 \%$ qui sont polygames. En effet, les jeunes hommes s'intéressaient plus à des activités non agricoles (petit commerce, orpaillage et autres prestations de services) et migrent vers les centres urbains laissant les activités agricoles au profit des plus âgés. Ces hommes manquaient de force physique nécessaire pour la conduite des opérations culturales. La majorité des répondants $(71 \%)$ sont analphabètes. En effet, $11,7 \%$ des enquêtés sont du niveau primaire et $0,8 \%$ au secondaire. L'alphabétisation en langue et l'école coranique occupaient respectivement $9,9 \%$ et $7,5 \%$ des répondants.

S'agissant des superficies cultivées, $65 \%$ des exploitants enquêtés avaient déclaré exploiter une superficie comprise entre 0,5 à 4,8 ha. Presque tous les exploitants enquêtés (92\%) cultivent le sorgho, maïs et le mil sur la moitié de leur terre. Le sorgho ou le mil sont souvent en association avec le niébé.

Performance économique et financière de production selon les différentes composantes de la technologie 'Microdose'. L'analyse des résultats des indicateurs montre que les composantes déterminent différemment la performance de chaque composante (Tableau 4). Les valeurs moyennes positives des différents indicateurs contribuaient à la valeur ajoutée et étaient par ailleurs économiquement et financièrement rentables. Cette contribution varie d'une composante à une autre pour les cultures de sorgho maïs et niébé mais reste invariante entre le mil et le sorgho.

En effet, l'adoption de la microdose générait une marge nette et un taux interne de rentabilité respectivement positifs $(\mathrm{MN}>0$ et $\mathrm{TRI}>$ au taux d'emprunt sur le marché) avec des valeurs 
TABLEAU 3. Caractéristiques socio-démographiques des chefs d'exploitation

\begin{tabular}{llrrr}
\hline Variables & Modalités & Kouritenga & Zondoma & Ensemble \\
\hline Genre (\%) & Homme & 79 & 69 & 73,9 \\
& Femme & 21 & 31 & 26,1 \\
Catégorie d'âges moyens (\%) & Age1: $<30$ ans & 2,8 & 2,8 & 2,8 \\
& Age2: 30 à 45 ans & 16,1 & 10,9 & 13,5 \\
& Age3: 46 à 65 ans & 24,8 & 27,3 & 26,1 \\
& Age4 :> 65 ans & 1,8 & 4,9 & 3,5 \\
Nombre d'actifs par ménage (moyenne) & & & & \\
& & $4,48(3,10)$ & $4,18(2,81)$ & $4,33(2,96)$ \\
Taille du ménage & & & & \\
& $1-7$ & 37,2 & 28,3 & 32,8 \\
& $8-10$ & 23,3 & 37,8 & 30,6 \\
Statut matrimonial & 11 et plus & 39,4 & 33,9 & 36,7 \\
& & & & \\
& Monogame & 58,3 & 42,2 & 50,3 \\
& Polygame & 37,8 & 56,1 & 46,9 \\
Groupement (\%) & Veuf(ve) & 3,3 & 1,1 & 2,2 \\
Formation technique (\%) & divorcé (e) & 0,6 & 0,6 & 0,6 \\
& Oui & 53,3 & 29,4 & 41,4 \\
& Oui & 26,7 & 19,4 & 23,1 \\
\hline
\end{tabular}

Source : Enquête socio-économique, 2014

( ) = écart-type

supérieures à celles des non adoptants. Ainsi, du point de vue de la marge nette et du taux interne de rentabilité, l'adoption de la microdose était rentable dans la zone de recherche.

Par ailleurs, la productivité moyenne de la main d'œuvre familiale (PML) et le ratio bénéfice-Coût (BC) montrent que la production du niébé et du sorgho était rentable mais l'adoption de la microdose est moins rentable (Tableau 4).

Le Tableau 5 indique les composantes de la technologie 'Microdose' présentant les meilleures valeurs des indicateurs de performance retenus. Il ressort des résultats d'analyse que la technologie 'Microdose (MD) avec Bandes enherbées (BE) avec Compost $(\mathrm{CO})$ ' présentait de meilleure performance du point de vue de la marge nette, du taux interne de rentabilité et du ratio bénéfice-coût.

De même, la composante MD + BE + Compost donnait la marge nette par ha la plus élevée (377 927 Fcfa), la composante MD +Zaï + F fumière donne la meilleure valorisation de la main d'œuvre familiale avec $8917 \mathrm{Fcfa} /$ $\mathrm{HJ}$ (Figs. 1 et 2). La meilleure efficacité économique était le paquet $\mathrm{MD}+\mathrm{BE}+$ Compost avec un Ratio Bénéfice sur Coût de 2,67 . Pour la valorisation du capital investi, $\mathrm{MD}+\mathrm{BE}+$ Compost avait le meilleur record évalué à $2,12 \%$. Ces indicateurs de performance n'ont pas révélé de différences significatives entre les deux localités.

Influence de la technologie 'Microdose' sur la performance économique et financière. Les résultats de la régression apparemment indépendante du Tableau 6 ont montré spécifiquement que les variations observées au niveau des variables explicatives éventuelles expliquent respectivement 14,70\%, 54,99\%, $43,54 \%$ et $42,78 \%$ des variations observées dans la performance économique et financière (respectivement pour la marge nette, la productivité moyenne du travail, le taux interne 
de rentabilité et le ratio bénéfice-coût). Ces variables explicatives sont constituées par la marge nette, la productivité moyenne du travail, le taux de rentabilité interne et le ratio bénéfice sur coût. Dans l'ensemble, les quatre (04) modèles étaient globalement significatifs au seuil de à $1 \%$ (Tableau 6). Le test d'indépendance de Breusch-Païen était significatif et traduit que la matrice de corrélation est significative $(P=0,000)$. On ne peut rejeter l'hypothèse nulle, les modèles sont liés entre eux. La modélisation simultanée utilisée dans le cadre de l'étude est donc bien justifiée.

La performance économique et financière de la technologie 'Microdose' était déterminée principalement par l'adoption de la microdose et les composantes de la technologie 'Microdose' (aménagement de cordon pierreux et utilisation du compost). D'autres facteurs avaient eu d'influence sur cette performance : il s'agit de l'âge du producteur, son appartenance à une association ou groupement, son sexe, son expérience en technique de microdose, la localité de l'exploitant et le capital variable de production. Ces facteurs sont significatifs et influencent négativement ou positivement les performances économiques des exploitations.

En effet, la composante "cordon pierreux", l'appartenance à des associations, le sexe, la localité Kouritenga, influençaient négativement la marge nette de production au seuil de 5\%. Par contre, l'âge de l'exploitant, l'expérience dans l'adoption de la microdose influençaient positivement au seuil de $1 \%$ respectivement et les charges variables de production au seuil de 5\% la marge nette de production.

Concernant la productivité moyenne de la main d'œuvre familiale, on avait enregistré les mêmes tendances que pour la marge nette. En revanche, la composante "cordon pierreux" (au seuil de 10\%), l'appartenance à des associations et la main d'œuvre totale (au seuil de $1 \%$ ), le sexe (au seuil de 5\%) influençaient négativement la performance économique des producteurs du point de vue de la productivité moyenne de la main d'œuvre familiale. 
TABLEAU 5. Performance des différentes technologies de la microdose

\begin{tabular}{llll}
\hline Indicateurs & Technologies & Moyenne & Test ANOVA \\
\hline Marge nette (F CFA/ha) & MD + BE + Compost & 377926,76 & $(\mathrm{~F}=0,697 ;$ ddl $=195 ; \mathrm{P}=0,726)$ \\
Productivité du travail (F CFA/hj) & MD + Zaï + Fumier & 8916,69 & $(\mathrm{~F}=0,278 ; \mathrm{ddl}=195 ; \mathrm{P}=0,985)$ \\
Taux de rentabilité interne (\%) & MD + BE + Compost & 2,12 & $(\mathrm{~F}=0,752 ; \mathrm{ddl}=195 ; \mathrm{P}=0,675)$ \\
Bénéfice sur Coût & MD + BE + Compost & 2,67 & $(\mathrm{~F}=0,514 ; \mathrm{ddl}=195 ; \mathrm{P}=0,879)$
\end{tabular}

Source : Enquête socio-économique, 2014

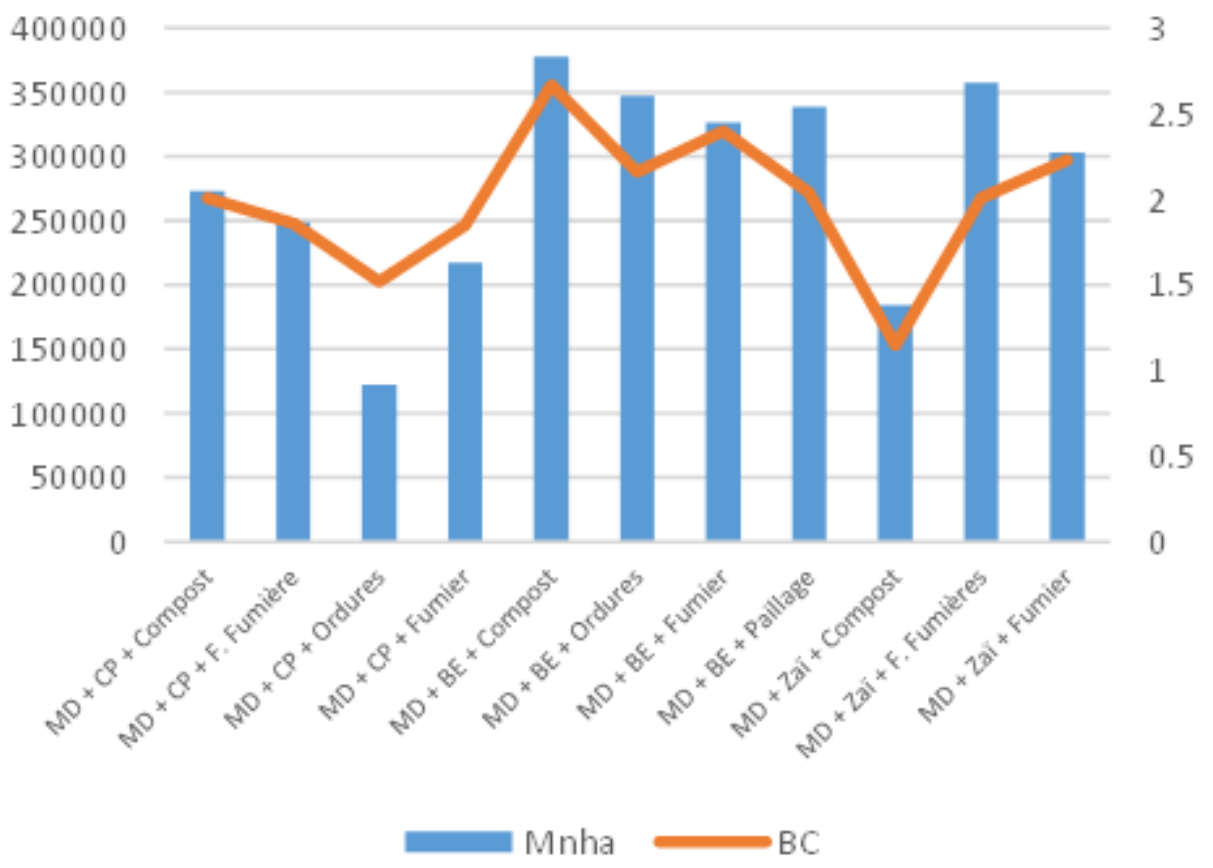

Figure 1. Bénéfice sur coût selon la composante technologie microdose.

Au niveau de la performance financière, le statut du chef d'exploitation et la localité Zondoma avaient un effet positif sur le taux de rentabilité interne respectivement au seuil de $5 \%$. Les capitaux de production (les charges fixes de production et la main d'œuvre totale) et l'appartenance à des associations ont un effet négatif sur le taux de rentabilité interne des exploitations au seuil de $1 \%$.

Concernant la rentabilité coût sur bénéfice, les facteurs dont le statut du chef d'exploitation et la localité Zondoma, avaient un effet positif sur le retour sur investissement à $5 \%$. Les charges fixes, l'appartenance à des associations et la main d'œuvre utilisée avaient un effet négatif sur le retour sur investissement au seuil de $1 \%$. Ces variables montraient des limites à l'adoption de la technologie 'Microdose'.

Les conditions agro-écologiques de la localité peuvent avoir une influence sur la productivité de l'exploitation. Les résultats de l'estimation font ressortir que la localité Zondoma ou Kouritenga affectait différemment les indicateurs de performances (marges nettes, la productivité moyenne de l'exploitation, le taux de rentabilité interne et le ratio coût-bénéfice). Ceci est serait lié aux 
Productivité du travail des composante et BC

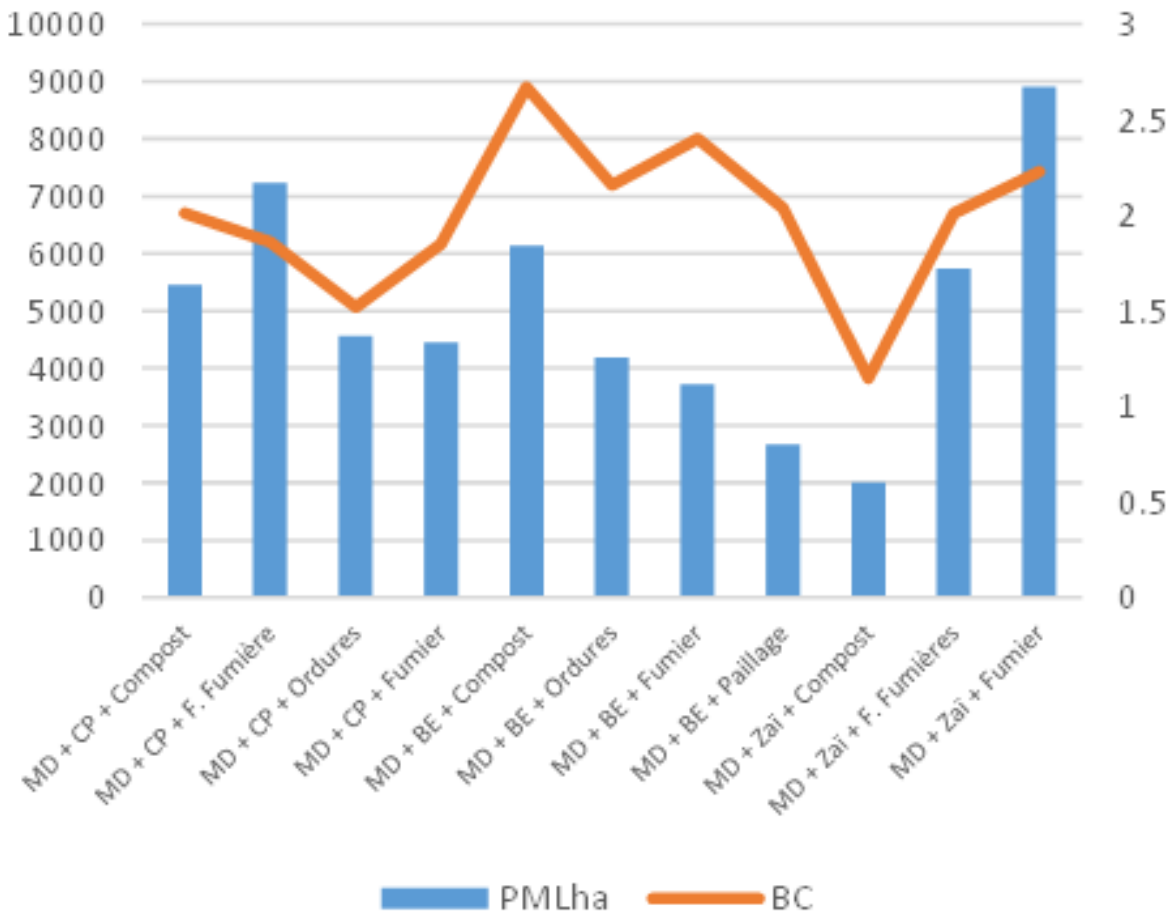

Figure 2. Productivité du travail selon les composantes de la technologie "Microdose".

facteurs spécifiques à chaque localité entre autres la pluviométrie, le niveau de dégradation des sols, etc.

Les facteurs significatifs à influences positives avaient des incidences qui galvanisaient la performance économique et financière contrairement aux facteurs déterminant négativement par la tendance des producteurs au peu d'intérêt à l'adoption de la technologie 'Microdose' en particulier.

\section{DISCUSSION}

Dans la région des savanes, les aléas climatiques et les activités humaines ont induit une crise de fertilité des sols, affectant les rendements agricoles et les revenus des ménages agricoles. C'est dans ce contexte que des pratiques agro-écologiques ont été vulgarisées auprès des paysans en vue d'intensifier la production agricole. La combinaison technique cordon pierreux et compost s'inscrit dans une logique de la conservation de la gestion de la fertilité du sol avec la technique de microdose en vue d'accroître le niveau de rendement du producteur à l'hectare.

De toute évidence, l'application de la fumure organique notamment du compost sur les parcelles de cultures a une influence positive sur les rendements et la productivité agricole. Dans la littérature empirique, des auteurs (Soule et al., 2000 ; Baffoe-Asare et al., 2013) ont démontré la contribution positive de la fumure organique sur le maintien du potentiel productif des terres de façon durable. Des auteurs (Dugué, 1994 ; Djiwagui et al., 2017) ont mis en évidence le fait que l'intensification agricole passe par le maintien de la fertilité des terres de cultures pour préserver le potentiel productif de façon durable.

Les contraintes qui freinent l'adoption conséquente de ces pratiques combinées sont principalement la faible disponibilité de la main 
TABLEAU 6. Résultats de l'estimation du modèle de régression à équations simultanées

\begin{tabular}{|c|c|c|c|c|c|c|c|c|}
\hline \multirow[t]{3}{*}{ Paramètres } & \multicolumn{2}{|c|}{ MN } & \multicolumn{2}{|c|}{ PML } & \multicolumn{2}{|c|}{ TRI } & \multicolumn{2}{|c|}{$\mathrm{B} / \mathrm{C}$} \\
\hline & Coefficients & $\mathrm{p}$ & Coefficients & $\mathrm{p}$ & Coefficients & $\mathrm{p}$ & Coefficient & $\mathrm{p}$ \\
\hline & \multicolumn{8}{|c|}{---------- Caractéristiques socio-démographiques } \\
\hline Age & $0,434(0,157)$ & $0,006 * *$ & $0,436(0,158)$ & $0,006 * * *$ & & & & \\
\hline Appartenance à une Association & $-0,401(0,097)$ & $0,000 * * *$ & $-0,401(0,09)$ & $0,000 * * *$ & $-0,484(0,171)$ & $0,005 * * *$ & $-0,492(0,170)$ & $0,004 * * *$ \\
\hline Statut matrimonial & $0,115(0,082)$ & 0,164 & $0,114(0,083)$ & 0,168 & $0,355(0,143)$ & $0,013 * *$ & $0,359(0,142)$ & $0,011 * *$ \\
\hline Sexe du producteur & $-0,192(0,087)$ & $0,027 * *$ & $-0,192(0,087)$ & $0,027 * *$ & & & & \\
\hline Formation technique agricole & & & & & $0,097(0,123)$ & 0,430 & $-0,260(0,167)$ & 0,118 \\
\hline Taille ménage & $-0,260(0,166)$ & 0,118 & $-0,260(0,167)$ & 0,118 & & & & \\
\hline Nombre d'actifs agricoles & $0,108(0,139)$ & 0,439 & $0,107(0,140)$ & 0,441 & $0,213(0,161)$ & 0,185 & $0,209(0,159)$ & 0,191 \\
\hline Expérience en microdose & $0,365(0,118)$ & $0,002 * * *$ & $0,365(0,118)$ & $0,002 * * *$ & $-0,146(0,207)$ & 0,481 & $-0,87(0,205)$ & 0,670 \\
\hline \multirow[t]{2}{*}{ Localité Zondoma } & - & - & $0,232(0,097)$ & $0,017 * *$ & $0,375(0,164)$ & $0,022 * *$ & $0,351(0,163)$ & $0,042 * *$ \\
\hline & \multicolumn{8}{|c|}{------------ Capitaux } \\
\hline Charges fixes & $-0,072(0,055)$ & 0,196 & $-0,745(0,055)$ & 0,183 & $-0,949(0,091)$ & $0,000 * * *$ & $-0,809(0,090)$ & $0,000 * * *$ \\
\hline Charges variables & $0,068(0,027)$ & $0,014 * *$ & $0,070(0,027)$ & $0,011^{* *}$ & & & & \\
\hline \multirow[t]{2}{*}{ Quantité de main d'œuvre totale } & $-0,094(0,052)$ & $0,074 *$ & $-0,094(0,053)$ & $0,000 * * *$ & $-0,598(0,094)$ & $0,000 * * *$ & $-0,94(0,053)$ & $0,000 * * *$ \\
\hline & \multicolumn{8}{|c|}{ 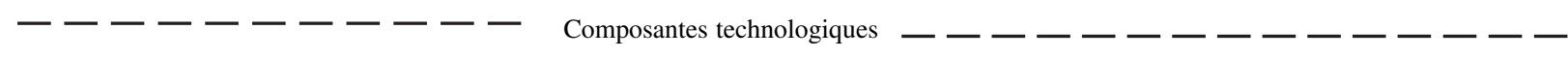 } \\
\hline Adoption de la microdose & $0,605(0,165)$ & $0,000 * * *$ & $0,605(0,165)$ & $0,000 * * *$ & & & & \\
\hline Tech. Cordon pierreux + Compost & $-0,187(0,104)$ & $0,073 *$ & $-0,187(0,104)$ & $0,075 * *$ & & & & \\
\hline Tech. Bande enherbée + Paillage & $0,012(0,042)$ & 0,765 & & & & & & \\
\hline Tech. Bande enherbée + Compost & $0,397(0,251)$ & 0,114 & $0,398(0,252)$ & 0,115 & $0,421(0,449)$ & 0,348 & $0,421(0,449)$ & 0,348 \\
\hline Tech. Cordon pierreux + fumier & & & & & $0,224(0,399)$ & 0,574 & $0,224(0,399)$ & 0,574 \\
\hline \multirow[t]{2}{*}{ Constante } & $10,588(0,875)$ & $0,000 * * *$ & $10,340(0,870)$ & $0,000 * * *$ & $13,203(1,153)$ & $0,000 * * *$ & $13,378(1,144)$ & $0,000 * * *$ \\
\hline & \multicolumn{8}{|c|}{ - - - - - - - Résumé du modèle } \\
\hline Observations & 329 & & 329 & & 329 & & 329 & \\
\hline Paramètres & 15 & & 14 & & 11 & & 11 & \\
\hline $\mathrm{R}$-square & 0,1470 & & 0,5499 & & 0,4354 & & 0,4278 & \\
\hline $\mathrm{Chi}^{2}$ & 71,14 & & 418,55 & & 263,69 & & 245,89 & \\
\hline Probabilité & 0,000 & & 0,000 & & 0,000 & & 0,000 & \\
\hline
\end{tabular}

Source : Résultats d'analyse des données, 2014 
d'œuvre, la pénibilité du travail. Certaines contraintes sont spécifiques aux pratiques comme le manque de pierres pour la construction des cordons pierreux. Somme toutes ces contraintes ont un effet négatif sur les indicateurs de rentabilité.

Par ailleurs, les facteurs sociodémographiques tels que l'âge du producteur, ont une influence significative positive sur les indicateurs de performance économique des exploitations agricoles marges nettes et la productivité de la main d'œuvre familiale mais sans impact significatif sur celui financier (le taux de rentabilité interne et le bénéfice sur coût). Les producteurs les plus âgés (adultes actifs et expérimentés) sont plus susceptibles à investir pour l'adoption afin d'améliorer les productions de sorgho mil maïs et niébé à long terme contrairement aux jeunes agriculteurs. En effet, dans la littérature des auteurs (Ngondjeb et al., 2014 ; Yabi et al., 2016) ont montré que les agriculteurs les plus âgés adopteraient plus facilement les innovations que les jeunes agriculteurs. On peut dire à cet effet, que les producteurs les plus âgés visent plus la valorisation pour des bénéfices dans la durabilité. Cependant, dans le cadre de l'agriculture de conservation, le rapport de la FAO (2003) estime que l'âge est un facteur « difficile à relier » à l'adoption de ce type d'agriculture dans la mesure où des études ont montré des résultats aussi bien positifs que négatifs.

De même, les chefs de ménages qui sont aussi les chefs d'exploitations sont généralement des hommes dans le monde agraire et dans la zone d'étude en particulier. Ils sont responsables de la gestion des exploitations notamment les mesures de durabilité économique de l'agriculture. Dans la zone de l'étude, les principales cultures sont le sorgho le mil le maïs et le niébé. L'accès à la terre est facilité pour ces derniers et leur rôle de chef de ménage garantissant ainsi la sécurité alimentaire et les besoins de toute la famille. Aussi bien les femmes que les hommes s'adaptent à leur manière pour une performance de leurs systèmes de cultures. Dans les exploitations agricoles familiales, ce sont les hommes qui ont un accès facile aux ressources (capital, terre, main d'œuvre), à l'information et à l'éducation ; trois facteurs primordiaux pour la performance économique des exploitations (Noma, 2011 ; Yabi et al., 2016 ; Tesfaye et al., 2016). Il est à retenir que, dans les exploitations agricoles de la zone d'étude, le fait d'être homme facilite le choix et l'adoption des stratégies adaptatives à la performance de l'exploitation.

Par la suite, l'expérience acquise en pratique de technologie 'Microdose' est un facteur déterminant la performance économique de l'exploitation agricole du point de vue de la marge nette et la productivité moyenne du travail. De tels résultats sont confirmés par les études de Abdulai et Huffman (2014) qui ont abouti à la conclusion selon laquelle, les expériences d'une innovation se font à travers ses caractéristiques telles que la profitabilité, l'efficacité et l'adaptabilité. Plus les agriculteurs ont une bonne expérience pratique de technologies agricoles, plus ils réalisent de meilleures performances dans leurs exploitations. Les travaux de Yegbemey et al. (2014) ont montré les performances de rendements agricoles des producteurs de maïs qui ont utilisé les semences améliorées pour s'adapter au changement climatique.

En accord avec tous ces auteurs, ce résultat contribue à confirmer l'hypothèse de l'analyse. Les producteurs ayant adopté la pratique de la microdose sur les cultures de sorgho, mil, maïs et du niébé améliorent leur production. Yabi et al. (2015) ont aussi montré dans leurs travaux les performances de la pratique de la microdose et du warrantage dans l'amélioration significative de la productivité du niébé dans cette même zone d'étude. Ces résultats rejoignent ceux de nombreuses études d'impact sur l'adoption technologies agricoles qui ont montré une augmentation significative de rendement des cultures (Assiya, 2010 ; Awotide et al., 2012 ; FAO, 2013 ; Maruod et 
al., 2013 ; Arouna et Diagne, 2013 ; Ogunniyi et Kehinde, 2015 ; Tesfaye et al., 2016).

Par ailleurs, la disponibilité des ressources d'investissement liées aux charges fixes, aux charges variables ou à la main d'œuvre familiale, sont des facteurs qui limitent l'adoption de la technologie. Cela se traduit dans notre étude par l'effet significatif et positif de cette variable sur la marge nette et sur la productivité moyenne du travail.

En effet, l'accès au capital suggère que des fonds soient alloués au producteur pour une meilleure gestion de son exploitation agricole et ce en vue d'augmenter son niveau de rendement. En effet des études ont montré que l'accès aux capitaux (fixe ou variable) est un facteur déterminant des exploitations agricoles (Roussy et al., 2015) pour de meilleures performances économiques. L'auteur a montré que plus les producteurs ont des charges fixes à rembourser par hectare de production de maïs, moins ils arrivent à tirer profit. Les charges d'aménagement des terres, l'acquisition des facteurs de production, toutes sont des facteurs qui agissent négativement sur le profit net du producteur.

Le capital variable néanmoins permet dans une certaine mesure de réaliser les combinaisons possibles des inputs pour d'obtenir un maximum d'output. De ce fait, l'effet positif de cette variable se fait remarquer sur la marge nette et sur la productivité moyenne du travail. Les conclusions de Meughoyi (2015) au Cameroun, les travaux de Maruod et al. (2013) au Soudan ont révélé que de meilleures productivités peuvent être enregistrées par les agriculteurs qui adoptent les nouvelles variétés. Dans cette optique, l'accès aux capitaux comme le soulignent déjà Madison (2006) et Deressa et al. (2009) permet au producteur d'adopter la technologie agricole pour mieux adapter son système cultural. La disponibilité du capital permet donc au producteur une meilleure optimisation de sa production. Cela se traduit dans notre étude par l'effet significatif et positif de cette variable sur la marge nette et sur la productivité moyenne du travail.

En effet, la corrélation entre la quantité de main d'œuvre totale utilisée et chacun des indicateurs de rentabilité utilisés est positive et significative. Cela est dû au fait qu'une augmentation indéfinie de la main d'œuvre aurait un effet négatif car tous les actifs agricoles ne pourront pas travailler dans les champs de maïs où même tous y travaillent mais la productivité ne permettrait pas de payer l'effort de tout le monde. Dans le ménage, il y a les autres enfants, vieillards et autres qui paraissent comme un déterminant négatif pour le producteur. Cette frange improductive affecte négativement la productivité et le taux de rentabilité interne agricole. Dans leurs études, Folefack et al. (2012) ont trouvé que la taille du ménage a un effet significatif et négatif sur la probabilité du choix de la stratégie. Ce résultat met ainsi en évidence que l'effectif du ménage peut être limitant par le coût supplémentaire de travail (Caffey et Kazmierczak, 1994 ; Batz et al., 1999; McBride et Daberkow, 2003 ), puisque l'effet attendu est la maximisation de la force de travail pour l'atteinte d'un rendement satisfaisant.

Enfin, un des objectifs spécifiques de cet article est de déterminer l'effet de la localité sur la productivité de l'exploitation. Il existe d'importantes variations dans les conditions locales qui nécessitent des réponses bien adaptées. Les producteurs ont des aspirations et des préoccupations liées à leurs caractères spécifiques selon la localité. Contrairement à cette situation, certains auteurs (Awotide et al., 2013 ; Ngendjeb et al., 2014) n'ont pas enregistré d'influence significative de la localité et quelques explications sont nécessaires. L'estimation a montré que la localité Kouritenga affecte négativement les marges nettes sont relatives aux charges consentis dans l'utilisation des intrants ainsi que celles charges liées aux opérations d'entretiens des cultures qui réduisent insidieusement les marges nettes 
dans cette localité. Le fait d'être dans la localité du Kouritenga les marges nettes sont affectées. Au Zondoma situé plus au Nord, la pluviosité est relativement plus faible et le niveau dégradation des sols est aussi plus élevé. Le travail (main d'œuvre familiale) et l'investissement dans la pratique de technologies de gestion de l'eau et de la fertilité dans la localité du Zondoma révèle des réponses remarquables qui contrastent avec la situation initiale. Les capitaux investis dans cette localité donc sont plus valorisés avec un meilleur taux de rentabilité et un bénéfice coût sur bénéfice au seuil de 5\%.

\section{CONCLUSION}

L'objectif de cette étude était d'évaluer l'effet de la technologie 'Microdose' sur les performances économiques des exploitations agricoles dans les provinces du Zondoma et du Kouritenga au Burkina Faso. Les indicateurs retenus ont montré des résultats positifs de toutes les composantes sur la performance économique et financière des exploitations agricoles. C'est dire qu'elles sont rentables. Les pratiques innovantes ou de conservation confirment davantage ces liens et la nouvelle technologie 'Microdose' de production est développée pour une amélioration de la performance économique des exploitations agricoles. Au niveau des localités, les producteurs font souvent des options de stratégies d'adaptation qui occasionnent souvent des coûts supplémentaires réduisant ainsi le profit net du producteur. Ces résultats suggèrent aux décideurs des politiques agricoles une prise en compte les différents facteurs d'influence significative dans le cadre de la promotion pour une mise à l'échelle de la technologie au profit des exploitants agricoles des localités similaires.

\section{REMERCIEMENTS}

L'équipe de chercheurs adresse ses remerciements au projet CRDI/ACDI
(l'Agence Canadienne de Développement International) $\mathrm{N}^{\circ}$ 106516-002 INuWaM «Intégration des Nutriments et de la Gestion de l'eau pour une Agriculture Durable au Sahel » financé par le Fonds Canadien de Recherche sur la Sécurité Alimentaire Internationale (FCRSAI), créé conjointement par le CRDI et l'Agence Canadienne de Développement International. Elle remercie également les autorités du développement rural des régions de l'Est et du Nord, les producteurs des provinces du Zondoma et du Kouritenga.

\section{REFERENCES}

Abossolo, S.A., Amougou, J.A. et Tchindjang, M. 2017. Perturbations climatiques et pratiques agricoles dans les zones agroécologiques du Cameroun: changements socio-économiques et problématique d'adaptation aux bouleversements climatiques. Editions Publibook. 24pp.

Amoussou, E., Vodounon, S.T., Hougni, A., Vissin, E.W., Houndenou, C., Mahe, G. et Boko, M., 2016. Changements environnementaux et vulnérabilité des écosystèmes dans le bassin-versant béninois du fleuve Niger. International Journal of Biological and Chemical Sciences 10: 2183-2201.

Arouna, A. et Diagne, A. 2013. Impact de la production de semence riz sur le rendement et le revenu des ménages agricoles : une étude de cas du Bénin. 4 th International Conference of the African Association of Agricultural Economists. Hammamet, Tunisia, 160547.

Assiya, A.C. 2010. Evaluation des impacts socio-économiques de l'utilisation des variétés améliorées (Mil HKP et Niébé TN578) au niveau ménages dans la région de Zinder : cas de Garagoumsa, Tirmini et Tanout au Niger. Mémoire d'Ingénieur Agronome. Institut Polytechnique Rural, Mali. 107pp. 
Awotide, B., Diagne, A. et Omonona, B. 2012. Impact of improved agricultural technology adoption on sustainable rice productivity and rural farmers' welfare in Nigeria: A local average treatment effect (Late) technique. African Economic Conference. Kigali, Rwanda. 23pp.

Djiwagui, Y., Yovo K., Adessou, S.K. et Agbenu K. 2017. Analyse socioeconomique des pratiques agroecologiques d'intensification de la production agricole dans la region des Savanes au Togo. Journal de la Recherche Scientifique de l'Université de Lomé 19 (2).

Dugué, P. 1994. Stratégies des producteurs et gestion des ressources naturelles en Afrique soudano-sahélienne. Les Cahiers de la Recherche développement $\mathrm{n}^{\circ} 39$.

Folefack, D.P., Sale, A. et Wakponou, A. 2012. Facteurs affectant l'utilisation de la fumure organique dans les exploitations agricoles en zone sahélienne du Cameroun. Afrique Science: Revue Internationale des Sciences et Technologie 8 (2):22-33.

Heckman, J. 2010. Building bridges between structural and program evaluation approaches to evaluating policy. Journal of Economic Literature 48(2):356-398.

Maruod, E., Elkhalil E. B., Elkhidir, B. and El Naim, M. 2013. Impact of improved seeds on small farmers productivity, income and livelihood in Umruwaba Locality of North Kordofan, Sudan. International Journal of Agriculture and Forestry 3(6):203-208.

Meughoyi, T.C. 2015. Semences améliorées et productivité agricole des exploitations familiales agricoles au Cameroun. Conférence sur «Accéléré la productivité agricole: la technologie et l'innovation, les actifs, l'accès au financement $\gg$ au Cameroun. pp. 1-13.

Ngendjeb, Y.D., Kamgnia, D.B., Nje, P. et Havard, M. 2014. L'évaluation économique de l'investissement dans la conservation des sols : le cas des aménagements antiérosifs dans le bassin versant du lac
Logdo au Cameroun. Canadian Journal of Agricultural Economics 62:393-410.

Ngondjeb, Y., Nje, P. et Havar, M. 2011. Déterminants de l'adoption des techniques de lutte contre l'érosion hydrique en zone cotonnière du Cameroun. Revue D'élevage et de Médecine Vétérinaire des Pays Tropicaux 64(1):9-19.

Ngendjeb, Y.D., Kamgnia, D.B., Nje, P. et Havard, M. 2014. L'évaluation économique de l'investissement dans la conservation des sols : le cas des aménagements antiérosifs dans le bassin versant du lac Logdo au Cameroun. Canadian Journal of Agricultural Economics 62:393-410.

Roussy, C., Ridier, A. et Chaib, K. 2015. Adoption d'innovations par les agriculteurs: Rôle des perceptions et des préférences. Working Paper SMARTLERECO N ${ }^{\circ}$ 15-03. https://www6.rennes. inra.fr/smart/ content/download/.../WP15-03.pdf consulté le 21 octobre 2015.

Sorel, K.D., Mesmin, T. et Casimir, N.P.I. 2017. Chapitre 13. Incidence de la variation pluviométrique sur les surfaces agricoles: stratégies d'adaptation paysanne à partir des savoir-faire endogènes à Bapa dans les hauts plateaux de l'Ouest-Cameroun. Perturbations Clim. Prat. Agric. Dans Zones Agroécologiques Cameroun Chang. Socio-Économiques Problématique Adapt. Aux Bouleversements Clim. 229pp.

Tesfaye, B., Bedada, B. and Mesay, Y. 2016. Impact of improved wheat technology adoption on productivity and income in Ethiopia. African Crop Science Journal 24 (Issue supplement S1): 127-135.

Yabi, J.A., Bachabi, F.X., Labiyi, I.A., Ode, C.A. et Ayena, R.L. 2016. Déterminants socio-économiques de l'adoption des pratiques culturales de gestion de la fetilité des sols utilisées dans la commune de Ouaké au Nord-Ouest du Bénin. International Journal of Biological and Chemical Sciences 10(2):779-792.

Yegbemey, R.N., Yabi, J.A., Aihounton, G.B. et Paraiso, A. 2014. Modélisation 
simultanée de la perception et de Zakari, S., 2017. Adoption des technologies l'adaptation au changement climatique : cas des producteurs de maïs du Nord Bénin (Afrique de l'Ouest). Cahier d'Agriculture 23(3):177-187.

et pratiques d'agriculture intelligente face au climat dans les sites CCAFS-(Niger), INRAN, Rapport final. 46pp. 